\title{
A Stable and High Order Accurate Conjugate Heat Transfer Problem
}

\author{
Jens Lindström \\ Uppsala University, Department of Information Technology, 751 05, Uppsala, Sweden \\ Jan Nordström \\ School of Mechanical, Industrial and Aeronautical Engineering \\ University of the Witvatersrand, PO WITS 2050, Johannesburg, South Africa \\ FOI, The Swedish Defence Research Agency, \\ Department of Aeronautics and Systems Integration, Stockholm, 164 90, Sweden \\ Uppsala University, Department of Information Technology, 751 05, Uppsala, Sweden
}

\begin{abstract}
This paper analyzes well-posedness and stability of a conjugate heat transfer problem in one space dimension. We study a model problem for heat transfer between a fluid and a solid. The energy method is used to derive boundary and interface conditions that make the continuous problem well-posed and the semi-discrete problem stable. The numerical scheme is implemented using 2nd, 3rd and 4th order finite difference operators on Summation-By-Parts (SBP) form. The boundary and interface conditions are implemented weakly. We investigate the spectrum of the spatial discretization to determine which type of coupling that gives attractive convergence properties. The rate of convergence is verified using the method of manufactured solutions.
\end{abstract}

\section{Introduction}

The coupling of fluid and heat equations is an area that has many interesting scientific and engineering applications. From the scientific side it is interesting to mathematically derive conditions to make the coupled system well-posed and compare with actual physics. The applications for conjugate heat transfer ranges between cooling of turbine blades, electronic components, nuclear reactors or spacecraft re-entry just to name a few. The particular application we are working towards here is a microscale satellite cold gas propulsion system with heat sources that will be used for controlling the flow rate [1]. See Figure 1.

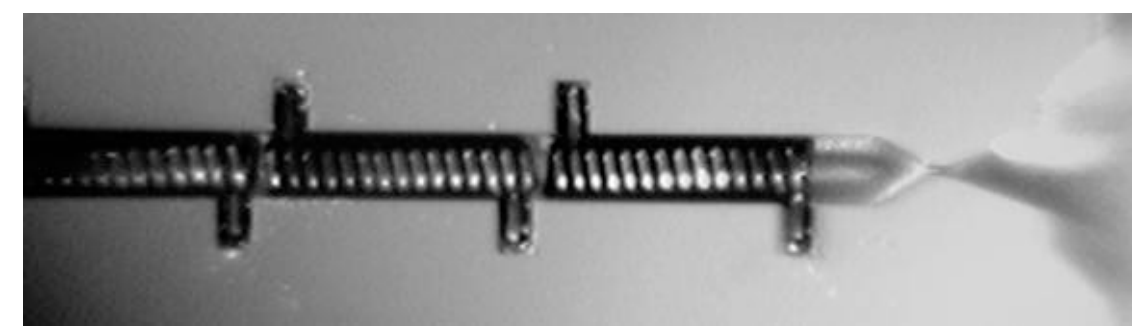

Figure 1: A micro machined nozzle with three heater coils positioned just before the nozzle throat. The nozzle throat is approximately $30 \mu \mathrm{m}$ in a heat exchange chamber. 
This paper is the first step of understanding the coupling procedure within our framework. The computational method that we are using is a finite difference method on Summation-By-Parts (SBP) form with the Simultaneous Approximation Term (SAT), a weak coupling at the fluid-solid interface. This method has been developed in many papers $[2,3,4,5,6,7]$ and used for many difficult problems where it has proven to be robust $[8,9,10,11]$. The extensions to multiple dimensions is relatively straightforward once the one-dimensional case has been investigated. The difficulty in extending to multiple dimensions lies rather in a high performance implementation than in the theory.

The main idea of the SBP and SAT framework is that the difference operators should mimic integration by parts in the continuous case. This framework makes the discrete equations closely related to the PDE:s themselves. The difference operators are constructed such that they shift to one-sided close to the boundaries. This results in an energy estimate which gives stability for a well-posed Cauchy problem. The SAT method implements the boundary conditions weakly and an energy estimate, and hence stability, can be obtained for a well-posed initial boundary value problem.

Since the operators shift to one-sided close to boundaries and interfaces there is no need to introduce ghost points or extrapolate values which in general causes stability issues. Once the scheme is correctly written and all coefficients determined the order of the scheme depends only on the order of the difference operators. In this paper we will present $2 \mathrm{nd}$, 3rd and 4 th order operators and study their performance. The details of these operators can be found in for example $[2,3,12]$.

\section{The continuous problem}

The equations we are studying in this paper are motivated by a gas flow in a long channel with heat sources. The channel is long compared to the height and hence the changes in the tangential direction are small in comparison to the changes in the normal direction, see Figure 2.

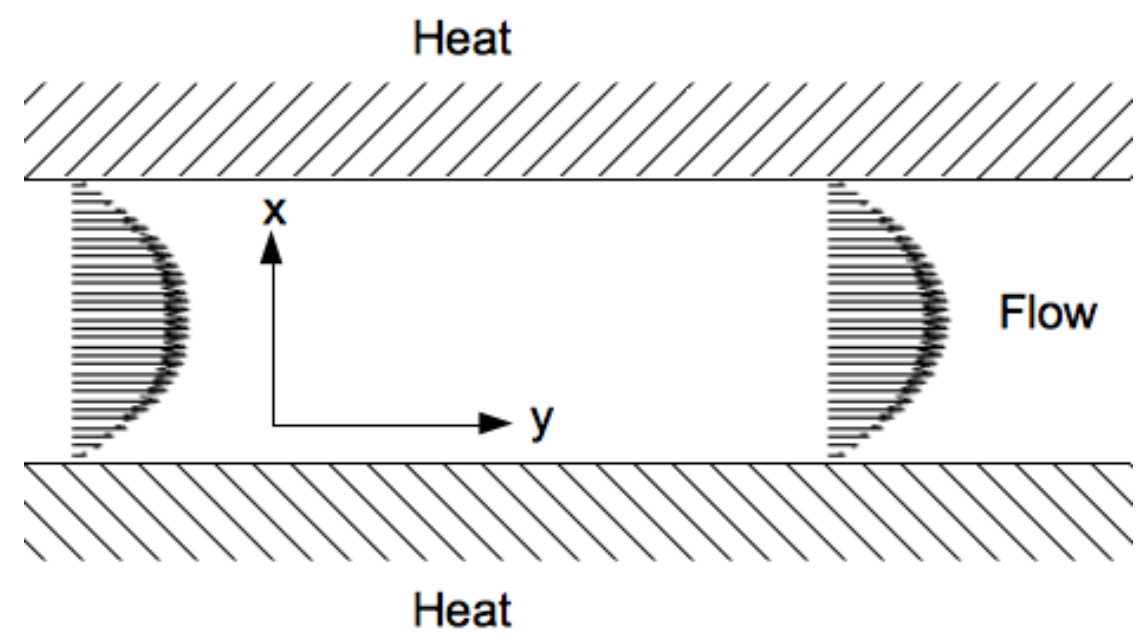

Figure 2: By assuming an infinitely long channel with homogenicity in the tangential direction $y$ we get a one-dimensional problem in the normal direction $x$ for the conjugate heat transfer problem

The equations are an incompletely parabolic system of equations for the flow and the 
scalar heat equation for the heat transfer,

$$
w_{t}+A w_{x}=\varepsilon B w_{x x}, \quad-1 \leq x \leq 0,
$$

and

where

$$
T_{t}=k T_{x x}, \quad 0 \leq x \leq 1,
$$

$$
w=\left[\begin{array}{l}
\rho \\
u \\
\mathcal{T}
\end{array}\right], \quad A=\left[\begin{array}{lll}
a & b & 0 \\
b & a & c \\
0 & c & a
\end{array}\right], \quad B=\left[\begin{array}{ccc}
0 & 0 & 0 \\
0 & \alpha & 0 \\
0 & 0 & \beta
\end{array}\right] .
$$

We can view (1) as the Navier-Stokes equations linearized and symmetrized around a constant state. In that case we would have

$$
a=\bar{u}, \quad b=\frac{\bar{c}}{\sqrt{\gamma}}, \quad c=\bar{c} \sqrt{\frac{\gamma-1}{\gamma}}, \quad \alpha=\frac{\lambda+2 \mu}{\bar{\rho}}, \quad \beta=\frac{\gamma \mu}{\operatorname{Pr} \bar{\rho}},
$$

where $\bar{u}, \bar{\rho}$ and $\bar{c}$ is the mean velocity, density and speed of sound. $\gamma$ is the ratio of specific heats, $\operatorname{Pr}$ the Prandtl number and $\lambda$ and $\mu$ are the second and dynamic viscosities, $[13,8,14]$. At this point the only assumption on the coefficients is that $\alpha, \beta>0$.

Our objective is to couple (1) and (2) at $x=0$ and investigate which boundary and interface conditions that will lead to a well-posed coupled system.

The number of boundary conditions at each boundary for (1) can be obtained by the Laplace transform technique [15]. Taking the Laplace transform in time of (1) gives us

$$
s \hat{w}+A \hat{w}_{x}=\varepsilon B \hat{w}_{x x}
$$

and by the ansatz $\hat{w}=\psi e^{\frac{k}{\varepsilon} x}$ we get

$$
\underbrace{\left(\tilde{s} I_{3}+k A-k^{2} B\right)}_{E(s, k)} \psi=0, \quad \tilde{s}=\epsilon s
$$

where $I_{3}$ is the $3 \times 3$ identity matrix. The polynomial degree in $k$ of $\operatorname{det}(E(s, k))$ gives the total number of boundary conditions needed and the sign of the roots as $\Re(s) \rightarrow \infty$ gives the number at each boundary [15]. By expanding $\operatorname{det}(E(s, k))$ we get

$$
\begin{aligned}
\operatorname{det}(E(s, k)) & =a \alpha \beta k^{5} \\
& +\left(\tilde{s} \alpha \beta+b^{2} \beta-a^{2} \beta-a^{2} \alpha\right) k^{4} \\
& +a\left(a^{2}-b^{2}-c^{2}-2 \tilde{s} \beta-2 \tilde{s} \alpha\right) k^{3} \\
& +\left(-\tilde{s}^{2} \beta+3 \tilde{s} a^{2}-\tilde{s} c^{2}-\tilde{s}^{2} \alpha-b^{2} \tilde{s}\right) k^{2} \\
& +3 a \tilde{s}^{2} k \\
& +\tilde{s}^{3}
\end{aligned}
$$

which is a $5^{\text {th }}$ degree polynomial in $k$ and hence (1) requires a total of five boundary conditions. To get the sign of the roots we let $k=c_{0} \tilde{s}^{\eta}$ which is inserted into (7). By letting $\Re(s) \rightarrow \infty$ we can balance the expression and see that $\eta=1$ and $\eta=\frac{1}{2}$ are the only possible choices. By inserting $\eta=1$ and $\eta=\frac{1}{2}$ into (7) we can compute all leading coefficients $c_{0}$ which are

$$
c_{0}=-\frac{1}{a}, \quad c_{0}= \pm \frac{1}{\sqrt{\alpha}}, \quad c_{0}= \pm \frac{1}{\sqrt{\beta}} .
$$

This gives the number of boundary conditions at each boundary summarized in Table 1 .

To simplify we assume for the rest of the paper that $a>0$. 


\begin{tabular}{|c|c|c|c|}
\hline & Total nr. of b.c. & At $x=-1$ & At $x=0$ \\
\hline$a>0$ & 5 & 3 & 2 \\
\hline$a=0$ & 4 & 2 & 2 \\
\hline$a<0$ & 5 & 2 & 3 \\
\hline
\end{tabular}

Table 1: Number of boundary conditions at each boundary depending on $a$

\subsection{Boundary conditions at $x=-1$}

Table 1 show that we need 3 boundary conditions at $x=-1$. The Laplace transform technique does however not say anything about the form of the boundary conditions required for a well-posed problem. To get the correct form we use the energy method [15].

Define the norm of $w$ as

$$
\|w\|^{2}=\int_{-1}^{0} w^{T} w d x
$$

By multiplying (1) with $w^{T}$ and integrating over the domain we get

$$
|| w \|_{t}^{2}=-\left.w^{T} A w\right|_{-1} ^{0}+\left.2 \varepsilon w^{T} B w_{x}\right|_{-1} ^{0}-2 \varepsilon \int_{-1}^{0} w_{x}^{T} B w_{x} d x .
$$

Since we are currently only interested in $x=-1$ we omit the right boundary terms and get $($ at $x=-1)$

$$
\|w\|_{t}^{2}=w^{T} A w-2 \varepsilon w^{T} B w_{x}-2 \varepsilon \int_{-1}^{0} w_{x}^{T} B w_{x} d x .
$$

Let

$$
X=\frac{1}{\sqrt{2} d}\left[\begin{array}{ccc}
-\sqrt{2} c & b & b \\
0 & d & d \\
\sqrt{2} b & c & c
\end{array}\right], \quad d=\sqrt{b^{2}+c^{2}},
$$

be the diagonalizing matrix of $A$. We have $X^{-1}=X^{T}$ and $A=X \Lambda X^{T}$ where

$$
\Lambda=\left[\begin{array}{ccc}
a & 0 & 0 \\
0 & a+d & 0 \\
0 & 0 & a-d
\end{array}\right]
$$

contains the eigenvalues of $A$. Using these relations we can write (11) as

$$
\|w\|_{t}^{2}=\left(X^{T} w\right)^{T} \Lambda\left(X^{T} w\right)-2 \varepsilon w^{T} B w_{x}-2 \varepsilon \int_{-1}^{0} w_{x}^{T} B w_{x} d x
$$

where

$$
X^{T} w=\frac{1}{\sqrt{2} d}\left[\begin{array}{c}
-\sqrt{2} c \rho+\sqrt{2} b \mathcal{T} \\
b \rho+d u+c \mathcal{T} \\
b \rho-d u+c \mathcal{T}
\end{array}\right]=\left[\begin{array}{l}
c_{1} \\
c_{2} \\
c_{3}
\end{array}\right]
$$

are the characteristic variables for the hyperbolic part. In order to bound the energy for the hyperbolic part we need to put boundary conditions on the characteristic variables that are related to the positive eigenvalues of $A$. If we assume that $a<d$ which corresponds to subsonic inflow, then $A$ has two positive eigenvalues and we need to use two boundary 
conditions on the corresponding characteristic variables. Thus we need to impose the boundary conditions

$$
\begin{aligned}
& c_{1}=\frac{1}{\sqrt{2} d}(-\sqrt{2} c \rho+\sqrt{2} b \mathcal{T})=f_{1}(t), \\
& c_{2}=\frac{1}{\sqrt{2} d}(b \rho+d u+c \mathcal{T})=f_{2}(t)
\end{aligned}
$$

to bound the hyperbolic part.

According to Table 1 we are allowed to use one more boundary conditions that will need to bound the parabolic term $-2 \varepsilon w^{T} B w_{x}$. Assume $f_{1}=f_{2}=0$. By taking linear combinations of (16) and (17) we can eliminate $\rho$ and obtain

$$
c u+d \mathcal{T}=0 .
$$

The parabolic term is expanded using relation (18) to obtain

$$
-2 \varepsilon w^{T} B w_{x}=-2 \varepsilon u\left(\alpha u_{x}-\frac{\beta c}{d} \mathcal{T}_{x}\right) .
$$

If we put

$$
\alpha d u_{x}-\beta c \mathcal{T}_{x}=f_{3}(t)
$$

as the final boundary condition for $(1)$ at $x=-1$, then with $f_{3}=0$ the parabolic term (19) is zero and all the boundary terms are bounded.

\subsection{Boundary conditions at $x=1$}

At $x=1$ we have the scalar heat equation. By applying the energy method we get

$$
\|T\|_{t}^{2}=2 k T T_{x}-2 k\left\|T_{x}\right\|^{2},
$$

from which it is easy to see that either

$$
T=h_{1}(t), \quad T_{x}=h_{2}(t) \quad \text { or } \quad \alpha_{1} T+\beta_{1} T_{x}=h_{3}(t)
$$

will result in an energy estimate (for suitable choices of the constants $\alpha_{1}$ and $\beta_{1}$ ). In the rest of the paper and in the numerical experiments we have used $T=h_{1}(t)$.

\subsection{Interface conditions at $x=0$}

At the interface we apply the energy method to both equations and add them together to get (when ignoring boundary terms)

$$
\frac{d}{d t}\left(\|w\|^{2}+\|T\|^{2}\right)=-w^{T} A w+2 \varepsilon w^{T} B w_{x}-2 k T T_{x}-2 \varepsilon \int_{-1}^{0} w_{x}^{T} B w_{x} d x-2 k \int_{0}^{1} T_{x}^{2} d x .
$$

Since we are considering the interface as a solid wall which separates the fluid from the solid and since we want a continuous heat transfer we impose

$$
u=0, \quad \mathcal{T}=T .
$$

Using the interface conditions (24), equation (23) reduces to

$$
\frac{d}{d t}\left(\|w\|^{2}+\|T\|^{2}\right)=2 \mathcal{T}\left(\beta \varepsilon \mathcal{T}_{x}-k T_{x}\right)-2 \varepsilon \int_{-1}^{0} w_{x}^{T} B w_{x} d x-2 k \int_{0}^{1} T_{x}^{2} d x
$$


and we can easily see that if we impose

$$
\beta \varepsilon \mathcal{T}_{x}-k T_{x}=0
$$

as the final interface condition we get an energy estimate. Without (26), the interface can act as an unphysical heat source or sink.

Using all these boundary and interface conditions we can conclude the following.

Proposition 2.1. Equations (1) and (2) coupled at $x=0$ are well-posed with boundary conditions (16), (17), (20) and (22) and interface conditions (24) and (26).

Remark 2.1. Note that in arriving at Proposition 2.1 we have assumed that the data is identically zero. If we had been able to obtain an energy estimate for non-zero data the problem would have been strongly well-posed [15].

Remark 2.2. No data has been assumed to be zero in the interface treatment. Hence the interface treatment can be called strongly well-posed.

\section{The semi-discrete problem}

Problem (1) is discretized on the single domain [-1,0] on a uniform grid of $M+1$ grid points. The vector $\mathbf{w}=\left[w_{0}, w_{1}, \ldots, w_{M}\right]^{T}=\left[\rho_{0}, u_{0}, \mathcal{T}_{0}, \rho_{1}, u_{1}, \mathcal{T}_{1}, \ldots, \rho_{M}, u_{M}, \mathcal{T}_{M}\right]^{T}$ is the discrete approximation of $w$. The derivatives are approximated by the operators on SBP form

$$
\begin{aligned}
\mathbf{w}_{x} & \approx\left(D_{1}^{L} \otimes I_{3}\right) \mathbf{w}=\left(P_{L}^{-1} Q_{L} \otimes I_{3}\right) \mathbf{w} \\
\mathbf{w}_{x x} & \approx\left(D_{2}^{L} \otimes I_{3}\right) \mathbf{w}=\left(P_{L}^{-1} Q_{L} \otimes I_{3}\right)^{2} \mathbf{w}
\end{aligned}
$$

where $P_{L}$ is a symmetric positive definite matrix and $Q_{L}$ is an almost skew symmetric matrix satisfying $Q_{L}+Q_{L}^{T}=B_{L}=\operatorname{diag}(-1,0, \ldots, 0,1)[2,3] . I_{3}$ is the $3 \times 3$ identity matrix. Problem (2) is similarly discretized on a uniform grid of $N+1$ grid points.

Remark 3.1. The approximation (28) has the drawback that the computational stencil is wide. Compact formulations that uses minimal bandwidth does however exist [3].

In (27) and (28) we have introduced the Kronecker product, defined as

$$
A \otimes B=\left[\begin{array}{ccc}
a_{11} B & \cdots & a_{1 n} B \\
\vdots & \ddots & \vdots \\
a_{m 1} B & \cdots & a_{m n} B
\end{array}\right]
$$

for the $m \times n$ and $p \times q$ matrices $A$ and $B$ respectively. It is a special case of a tensor product so it is bilinear and associative. Some of its important properties are

$$
\begin{aligned}
(A \otimes B)(C \otimes D) & =(A C \otimes B D), \\
(A \otimes B)^{-1} & =\left(A^{-1} \otimes B^{-1}\right)
\end{aligned}
$$

if the usual matrix products and inverses are defined.

With these tools and the boundary and interface conditions derived in Proposition 2.1 we can discretize (1) and (2) using the SAT method as 


$$
\begin{aligned}
\mathbf{w}_{t}= & -\left(D_{1}^{L} \otimes A\right) \mathbf{w}+\varepsilon\left(D_{2}^{L} \otimes B\right) \mathbf{w} \\
& +\left(P_{L}^{-1} E_{0}^{L} \otimes \Sigma_{1}^{0}\right)\left(X^{T} w_{0}-g_{1}^{0}\right) \\
& +\left(P_{L}^{-1} E_{0}^{L} \otimes \Sigma_{3}^{0}\right)\left(\alpha d\left(D_{1}^{L} u\right)_{0}-\beta c\left(D_{1}^{L} \mathcal{T}\right)_{0}-g_{3}^{0}\right) \\
& +\left(P_{L}^{-1}\left(D_{1}^{L}\right)^{T} E_{0}^{L} \otimes \Sigma_{5}^{0}\right)\left(c u_{0}+d \mathcal{T}_{0}-g_{5}^{0}\right) \\
& +\left(P_{L}^{-1} E_{M}^{L} \otimes \Sigma_{1}^{M}\right)\left(w_{M}-g_{1}^{M}\right) \\
& +\left(P_{L}^{-1} E_{M}^{L} \otimes \Sigma_{2}^{M}\right)\left(w_{M}-g_{1}^{M}\right) \\
& +\left(P_{L}^{-1} E_{M}^{L} \otimes \Sigma_{3}^{M}\right)\left(\mathcal{T}_{M}-T_{0}\right) \\
& +\left(P_{L}^{-1}\left(D_{1}^{L}\right)^{T} E_{M}^{L} \otimes \Sigma_{4}^{M}\right)\left(\mathcal{T}_{M}-T_{0}\right) \\
& +\left(P_{L}^{-1} E_{M}^{L} \otimes \Sigma_{5}^{M}\right)\left(\beta \varepsilon\left(D_{1}^{L} \mathcal{T}\right)_{M}-k\left(D_{1}^{R} T\right)_{0}\right) \\
& -\mathrm{DI}_{L} \\
\mathbf{T}_{t}= & k D_{2}^{R} \mathbf{T} \\
& +\tau_{1}^{0} P_{R}^{-1} E_{0}^{R}\left(T_{0}-\mathcal{T}_{M}\right) \\
& +\tau_{2}^{0} P_{R}^{-1}\left(D_{1}^{R}\right)^{T} E_{0}^{R}\left(T_{0}-\mathcal{T}_{M}\right) \\
& +\tau_{3}^{0} P_{R}^{-1} E_{0}^{R}\left(k\left(D_{1}^{R} T\right)_{0}-\beta \varepsilon\left(D_{1}^{L} \mathcal{T}\right)_{M}\right) \\
& +\tau_{1}^{N} P_{R}^{-1} E_{N}^{R}\left(T_{N}-h_{1}^{N}\right) \\
& -\mathrm{DI}_{R} .
\end{aligned}
$$

The matrices $E_{0}^{L}=\operatorname{diag}(1,0, \ldots, 0), E_{M}^{L}=\operatorname{diag}(0, \ldots, 0,1)$ and $E_{0, N}^{R}$ similarly defined, are used to select boundary elements. The $3 \times 3$ matrices $\Sigma_{i}^{0, M}$ and coefficients $\tau_{j}^{0, N}$ are called penalty matrices and penalty coefficients which have to be determined for stability $[2,3,4]$. The last term in (32) and (33) are artificial dissipation operators which reduces spurious oscillations. An extensive study of these operators can be found in [12]. The details are beyond the scope of this paper. They do not cause stability or accuracy problems and will be assumed to be treated correctly in the rest of the paper, and hence omitted in the stability derivations to keep the notation as simple as possible.

Remark 3.2. In (32) we have $X^{T} w_{0}-g_{1}^{0}=\left[c_{1}-f_{1}, c_{2}-f_{2}, c_{3}-f_{3}\right]^{T}$ where $c_{1}, c_{2}$ and $c_{3}$ are the characteristic variables. Moreover $w_{M}-g_{1}^{M}=\left[\rho_{M}-g_{1}, u_{M}-g_{2}, \mathcal{T}_{M}-g_{3}\right]^{T}$. The rest of the SAT boundary and interface terms are $3 \times 1$ vectors with the scalar values given on each row. The penalty matrices are constructed such that they select the correct entries and cancels the rest.

\subsection{Stability conditions at $x=-1$}

Consider the semi-discrete scheme (32) at the left boundary $x=-1$,

$$
\begin{aligned}
\mathbf{w}_{t}= & -\left(D_{1}^{L} \otimes A\right) \mathbf{w}+\varepsilon\left(D_{2}^{L} \otimes B\right) \mathbf{w} \\
& +\left(P_{L}^{-1} E_{0}^{L} \otimes \Sigma_{1}^{0}\right)\left(X^{T} w_{0}-g_{1}^{0}\right) \\
& +\left(P_{L}^{-1} E_{0}^{L} \otimes \Sigma_{3}^{0}\right)\left(\alpha d\left(D_{1}^{L} u\right)_{0}-\beta c\left(D_{1}^{L} \mathcal{T}\right)_{0}-g_{3}^{0}\right) \\
& +\left(P_{L}^{-1}\left(D_{1}^{L}\right)^{T} E_{0}^{L} \otimes \Sigma_{5}^{0}\right)\left(c u_{0}+d \mathcal{T}_{0}-g_{5}^{0}\right) .
\end{aligned}
$$

The penalty terms related to the interface are omitted. We will use the discrete analogue of the energy method to show that the scheme is stable. Define the discrete norm

$$
\|\mathbf{w}\|_{P_{L}}^{2}=\mathbf{w}^{T}\left(P_{L} \otimes I_{3}\right) \mathbf{w}
$$


where $I_{3}$ is the $3 \times 3$ identity matrix, and premultiply (34) with $\mathbf{w}^{T}\left(P \otimes I_{3}\right)$. Since $D_{1}^{L}$ and $D_{2}^{L}$ are on SBP form we obtain after some algebra

$$
\begin{aligned}
\frac{d}{d t}\|\mathbf{w}\|_{P_{L}}^{2} & =w_{0}^{T} A w_{0}-2 \varepsilon w_{0}^{T} B\left(D_{1}^{L} w\right)_{0}-2 \varepsilon\left(D_{1}^{L} \mathbf{w}\right)^{T}\left(I_{N+1} \otimes B\right)\left(D_{1}^{L} \mathbf{w}\right) \\
& +2 w_{0}^{T} \Sigma_{1}^{0}\left(X^{T} w_{0}-g_{1}^{0}\right) \\
& +2 w_{0}^{T} \Sigma_{3}^{0}\left(\alpha d\left(D_{1}^{L} u\right)_{0}-\beta c\left(D_{1}^{L} \mathcal{T}\right)_{0}-g_{3}^{0}\right) \\
& +2\left(D_{1}^{L} w\right)_{0}^{T} \Sigma_{5}^{0}\left(c u_{0}+d \mathcal{T}_{0}-g_{5}^{0}\right) .
\end{aligned}
$$

As in the continuous case we let $g_{1}^{0}=g_{3}^{0}=g_{5}^{0}=0$ and consider the hyperbolic and parabolic parts separately.

The hyperbolic part with the corresponding penalty term is

$$
w_{0}^{T} A w_{0}+2 w_{0}^{T} \Sigma_{1}^{0} X^{T} w_{0}
$$

and we need to choose $\Sigma_{1}^{0}$ such that (37) is negative semi-definite. Since $X^{-1}=X^{T}$ we can rewrite (37) as

$$
w_{0}^{T} A w_{0}+2 w_{0}^{T} \Sigma_{1}^{0} X^{T} w_{0}=\left(X^{T} w_{0}\right)^{T}\left(\Lambda+2 X^{T} \Sigma_{1}^{0}\right)\left(X^{T} w_{0}\right)
$$

and by letting $X^{T} \Sigma_{1}^{0}=\tilde{\Sigma}_{1}^{0}=\operatorname{diag}\left(\sigma_{1}^{0}, \sigma_{2}^{0}, 0\right)$ with

$$
a+2 \sigma_{1}^{0} \leq 0, \quad a+d+2 \sigma_{2}^{0} \leq 0
$$

we have

$$
w_{0}^{T} A w_{0}+2 w_{0}^{T} \Sigma_{1}^{0} X^{T} w_{0} \leq 0 .
$$

Thus the penalty matrix related to the hyperbolic part is

$$
\Sigma_{1}^{0}=X \tilde{\Sigma}_{1}^{0}=\frac{1}{\sqrt{2} d}\left[\begin{array}{ccc}
-\sqrt{2} c \sigma_{1}^{0} & b \sigma_{2}^{0} & 0 \\
0 & d \sigma_{2}^{0} & 0 \\
\sqrt{2} b \sigma_{1}^{0} & c \sigma_{2}^{0} & 0
\end{array}\right] .
$$

where $\sigma_{1}^{0}$ and $\sigma_{2}^{0}$ are appropriately bounded in (39).

The parabolic part with the corresponding penalty terms is

$$
-2 \varepsilon w_{0}^{T} B\left(D_{1}^{L} w\right)_{0}+2 w_{0}^{T} \Sigma_{3}^{0}\left(\alpha d\left(D_{1}^{L} u\right)_{0}-\beta c\left(D_{1}^{L} \mathcal{T}\right)_{0}\right)+2\left(D_{1}^{L} w\right)_{0}^{T} \Sigma_{5}^{0}\left(c u_{0}+d \mathcal{T}_{0}\right)
$$

and again we have to choose $\Sigma_{3}^{0}$ and $\Sigma_{5}^{0}$ such that (42) is negative semi-definite. Let

$$
\Sigma_{3}^{0}=\left[\begin{array}{ccc}
0 & 0 & 0 \\
0 & \varepsilon \sigma_{3}^{0} & 0 \\
0 & 0 & \varepsilon \sigma_{4}^{0}
\end{array}\right], \quad \Sigma_{5}^{0}=\left[\begin{array}{ccc}
0 & 0 & 0 \\
0 & \varepsilon \sigma_{5}^{0} & 0 \\
0 & 0 & \varepsilon \sigma_{6}^{0}
\end{array}\right] .
$$

We formulate (42) as a quadratic form $\varepsilon v_{0}^{T} M_{0} v_{0}$ with $v_{0}=\left[u_{0},\left(D_{1}^{L} u\right)_{0}, \mathcal{T}_{0},\left(D_{1}^{L} \mathcal{T}\right)_{0}\right]^{T}$ and

$$
M_{0}=\left[\begin{array}{cccc}
0 & -\alpha+\alpha d \sigma_{3}^{0}+c \sigma_{5}^{0} & 0 & -\beta c \sigma_{3}^{0}+c \sigma_{6}^{0} \\
-\alpha+\alpha d \sigma_{3}^{0}+c \sigma_{5}^{0} & 0 & \alpha d \sigma_{4}^{0}+d \sigma_{5}^{0} & 0 \\
0 & \alpha d \sigma_{4}^{0}+d \sigma_{5}^{0} & 0 & -\beta-\beta c \sigma_{4}^{0}+d \sigma_{6}^{0} \\
-\beta c \sigma_{3}^{0}+c \sigma_{6}^{0} & 0 & -\beta-\beta c \sigma_{4}^{0}+d \sigma_{6}^{0} & 0
\end{array}\right] .
$$

In order for (42) to be negative semi-definite we need to choose the coefficients $\sigma_{i}^{0}$ such that $M_{0} \leq 0$. Since all diagonal entries of $M_{0}$ is zero, all other entries must also be zero. This results in a system of equations with a one parameter family of solutions

$$
r \in \mathbb{R}, \quad \sigma_{3}^{0}=\frac{1+c r}{d}, \quad \sigma_{4}^{0}=r, \quad \sigma_{5}^{0}=-\alpha r, \quad \sigma_{6}^{0}=\frac{\beta(1+c r)}{d} .
$$


With these choices $M_{0}=0$ and we obtain an energy estimate where

$$
\frac{d}{d t}\|\mathbf{w}\|_{P_{L}}^{2} \leq-2 \varepsilon\left(D_{1}^{L} \mathbf{w}\right)^{T}\left(I_{N+1} \otimes B\right)\left(D_{1}^{L} \mathbf{w}\right) \leq 0
$$

and hence the left boundary is stable.

\subsection{Stability conditions at $x=1$}

Consider the semi-discrete scheme (33) at $x=1$

$$
\mathbf{T}_{t}=k D_{2}^{R} \mathbf{T}+\tau_{1}^{N} P_{R}^{-1} E_{N}^{R}\left(T_{N}-h_{1}^{N}\right) .
$$

By assuming $h_{1}^{N}=0$ and premultiplying with $\mathbf{T}^{\mathbf{T}} P_{R}$ we get (when ignoring interface terms)

$$
\frac{d}{d t}\|\mathbf{T}\|_{P_{R}}^{2}=2 k T_{N}\left(D_{1}^{R} T\right)_{N}+2 \tau_{1}^{N} T_{M}^{2}-2 k\left(D_{1}^{R} \mathbf{T}\right)^{T} P_{R}\left(D_{1}^{R} \mathbf{T}\right)
$$

and by choosing

$$
\tau_{1}^{N} \leq \frac{-k}{4 p_{N}^{R}}
$$

where $p_{N}^{R} \sim 1 / \Delta x_{R}$ we have an energy estimate which implies stability for the right boundary, see the derivation of (66). More details on how to obtain $\tau_{1}^{M}$ can be found in $[5,6]$.

\subsection{Stability conditions at $x=0$}

At $x=0$ we have the two interface schemes

$$
\begin{aligned}
\mathbf{w}_{t}= & -\left(D_{1}^{L} \otimes A\right) \mathbf{w}+\varepsilon\left(D_{2}^{L} \otimes B\right) \mathbf{w} \\
& +\left(P^{-1} E_{M}^{L} \otimes \Sigma_{1}^{M}\right)\left(w_{M}-g_{1}^{M}\right) \\
& +\left(P^{-1} E_{M}^{L} \otimes \Sigma_{2}^{M}\right)\left(w_{M}-g_{1}^{M}\right) \\
& +\left(P^{-1} E_{M}^{L} \otimes \Sigma_{3}^{M}\right)\left(\mathcal{T}_{M}-T_{0}\right) \\
& +\left(P^{-1}\left(D_{1}^{L}\right)^{T} E_{M}^{L} \otimes \Sigma_{4}^{M}\right)\left(\mathcal{T}_{M}-T_{0}\right) \\
& +\left(P^{-1} E_{M}^{L} \otimes \Sigma_{5}^{M}\right)\left(\beta \varepsilon\left(D_{1}^{L} \mathcal{T}\right)_{M}-k\left(D_{1}^{R} T\right)_{0}\right) \\
\mathbf{T}_{t}= & k D_{2}^{R} \mathbf{T} \\
& +\tau_{1}^{0} P_{R}^{-1} E_{0}^{R}\left(T_{0}-\mathcal{T}_{M}\right) \\
& +\tau_{2}^{0} P_{R}^{-1}\left(D_{1}^{R}\right)^{T} E_{0}^{R}\left(T_{0}-\mathcal{T}_{M}\right) \\
& +\tau_{3}^{0} P_{R}^{-1} E_{0}^{R}\left(k\left(D_{1}^{R} T\right)_{0}-\beta \varepsilon\left(D_{1}^{L} \mathcal{T}\right)_{M}\right) .
\end{aligned}
$$

The penalty terms related to the outer boundares are omitted. 
A formulation which clearly shows the coupled system can be written

$$
\begin{aligned}
{\left[\begin{array}{c}
\mathbf{w} \\
\mathbf{T}
\end{array}\right]_{t} } & =\left[\begin{array}{cc}
D_{1}^{L} \otimes(-A) & 0 \\
0 & 0
\end{array}\right]\left[\begin{array}{c}
\mathbf{w} \\
\mathbf{T}
\end{array}\right]+\left[\begin{array}{cc}
D_{1}^{L} \otimes \epsilon B & 0 \\
0 & k D_{2}^{R}
\end{array}\right]\left[\begin{array}{c}
\mathbf{w} \\
\mathbf{T}
\end{array}\right] \\
& +\bar{P}^{-1} \underbrace{\left[\begin{array}{c}
e_{M}^{L} \otimes \tilde{\Sigma}_{3}^{M} \\
-\tau_{1}^{0} e_{0}^{R}
\end{array}\right]\left[\begin{array}{c}
e_{M}^{L} \otimes f_{3} \\
-e_{0}^{R}
\end{array}\right]}_{J_{1}}\left[\begin{array}{c}
\mathbf{w} \\
\mathbf{T}
\end{array}\right] \\
& +\bar{P}^{-1} \underbrace{\left[\begin{array}{c}
\left(D_{1}^{L}\right)^{T} e_{M}^{L} \otimes \tilde{\Sigma}_{4}^{M} \\
-\tau_{2}^{0}\left(D_{1}^{R}\right)^{T} e_{0}^{R}
\end{array}\right]\left[\begin{array}{c}
e_{M}^{L} \otimes f_{3} \\
-e_{0}^{R}
\end{array}\right]^{T}\left[\begin{array}{c}
\mathbf{w} \\
\mathbf{T}
\end{array}\right]}_{J_{2}} \\
& +\bar{P}^{-1} \underbrace{\left[\begin{array}{c}
e_{M}^{L} \otimes \tilde{\Sigma}_{5}^{M} \\
-\tau_{3}^{0} e_{0}^{R}
\end{array}\right]\left[\begin{array}{c}
\beta \epsilon\left(D_{1}^{L} \otimes I_{3}\right)^{T}\left(e_{M}^{L} \otimes f_{3}\right) \\
-k\left(D_{1}^{R}\right)^{T} e_{0}^{R}
\end{array}\right]^{T}}_{J_{3}}\left[\begin{array}{c}
\mathbf{w} \\
\mathbf{T}
\end{array}\right]
\end{aligned}
$$

where

$$
\bar{P}^{-1}=\left[\begin{array}{cc}
P_{L}^{-1} \otimes I_{3} & 0 \\
0 & P_{R}^{-1}
\end{array}\right], \quad \tilde{\Sigma}_{i}^{M}=\left[0,0, \sigma_{i}^{M}\right]^{T}, \quad f_{3}=[0,0,1]^{T} .
$$

The interface matrices $J_{i}$ are sparse with entries only close to the interface. For 2nd order difference operators they are

$$
\begin{aligned}
& J_{1}=\left[\begin{array}{cccccc}
0 & & \cdots & \cdots & & 0 \\
\vdots & & \vdots & \vdots & & \vdots \\
& \cdots & \sigma_{3}^{M} & -\sigma_{3}^{M} & \cdots & \\
& \cdots & -\tau_{1}^{0} & \tau_{1}^{0} & \cdots & \\
\vdots & & \vdots & \vdots & & \vdots \\
0 & & \cdots & \cdots & & 0
\end{array}\right] \\
& J_{2}=\left[\begin{array}{cccccc}
0 & & \cdots & \cdots & & 0 \\
\vdots & & \vdots & \vdots & & \vdots \\
& \cdots & -\frac{\sigma_{4}^{M}}{\Delta x_{L}} & \frac{\sigma_{4}^{M}}{\Delta x_{L}} & \cdots & \\
& \cdots & 0 & 0 & \cdots & \\
\cdots & 0 & 0 & \cdots & \\
& \cdots & \frac{\sigma_{4}^{M}}{\Delta x_{L}} & -\frac{\sigma_{4}^{M}}{\Delta x_{L}} & \cdots & \\
& \cdots & -\frac{\tau_{2}^{0}}{\Delta x_{R}} & \frac{\tau_{2}^{0}}{\Delta x_{L}} & \cdots & \\
& \cdots & \frac{\tau_{2}^{0}}{\Delta x_{R}} & -\frac{\tau_{2}^{0}}{\Delta x_{L}} & \cdots & \\
\vdots & & \vdots & \vdots & & \vdots \\
0 & & \cdots & \cdots & & 0
\end{array}\right], \\
& J_{3}=\left[\begin{array}{cccccccccc}
0 & & & & \cdots & \cdots & & & & 0 \\
\vdots & & & & \vdots & \vdots & & & & \vdots \\
& \cdots & -\frac{\sigma_{5}^{M} \beta \epsilon}{\Delta x_{L}} & 0 & 0 & \frac{\sigma_{5}^{M} \beta \epsilon}{\Delta x_{L}} & -\frac{\sigma_{5}^{M} \beta \epsilon}{\Delta x_{L}} & \frac{\sigma_{5}^{M} \beta \epsilon}{\Delta x_{L}} & \cdots & \\
& \cdots & -\frac{\tau_{3}^{0} k}{\Delta x_{R}} & 0 & 0 & \frac{\tau_{3}^{0} k}{\Delta x_{R}} & -\frac{\tau_{3}^{0} k}{\Delta x_{R}} & \frac{\tau_{3}^{0} k}{\Delta x_{R}} & \cdots & \\
\vdots & & & & \vdots & \vdots & & & & \vdots \\
0 & & & & \cdots & \cdots & & & & 0
\end{array}\right]
\end{aligned}
$$

By letting $g_{1}^{M}=0$, applying the energy method to both equations and adding together 
we get (when ignoring the outer boundary terms)

$$
\begin{aligned}
\frac{d}{d t}\left(\|\mathbf{w}\|_{P_{L}}^{2}+\|\mathbf{T}\|_{P_{R}}^{2}\right) & =-w_{M}^{T} A w_{M}+2 \varepsilon w_{M}^{T} B\left(D_{1}^{L} w\right)_{M}-2 \varepsilon\left(D_{1}^{L} \mathbf{w}\right)^{T}\left(I_{N} \otimes B\right)\left(D_{1}^{L} \mathbf{w}\right) \\
& +2 w_{M}^{T} \Sigma_{1}^{M} w_{M}+2 w_{M}^{T} \Sigma_{2}^{M} w_{M} \\
& +2 w_{M}^{T} \Sigma_{3}^{M}\left(\mathcal{T}_{M}-T_{0}\right)+\left(D_{1}^{L} w\right)_{N}^{T} \Sigma_{4}^{M}\left(\mathcal{T}_{M}-T_{0}\right) \\
& +2 w_{M}^{T} \Sigma_{5}^{M}\left(\beta \varepsilon\left(D_{1}^{L} w\right)_{M}-k\left(D_{1}^{R} T\right)_{0}\right) \\
& -2 k T_{0}\left(D_{1}^{R} T\right)_{0}-2 k\left(D_{1}^{R} \mathbf{T}\right)^{T} P_{R}\left(D_{1}^{R} \mathbf{T}\right) \\
& +2 \tau_{1}^{0} T_{0}\left(T_{0}-\mathcal{T}_{M}\right)+2 \tau_{2}^{0}\left(D_{1}^{R} T\right)_{0}\left(T_{0}-\mathcal{T}_{M}\right) \\
& +2 \tau_{3}^{0} T_{0}\left(k\left(D_{1}^{R} T\right)_{0}-\beta \varepsilon\left(D_{1}^{L} \mathcal{T}\right)_{M}\right)
\end{aligned}
$$

As in the continuous case we have the hyperbolic part with the corresponding penalty term

$$
-w_{M}^{T} A w_{M}+2 w_{M}^{T} \Sigma_{1}^{M} w_{M}=w_{M}^{T}(\underbrace{-A+2 \Sigma_{1}^{M}}_{M_{H}}) w_{M}
$$

which we want to bound by making $M_{H}$ negative semi-definite. Note that $A$ is symmetric by assumtion. By choosing

$$
\Sigma_{1}^{M}=\left[\begin{array}{ccc}
0 & \sigma_{1}^{H} & 0 \\
0 & \sigma_{2}^{H} & 0 \\
0 & \sigma_{3}^{H} & 0
\end{array}\right]
$$

we can explicitly compute the eigenvalues of $M_{H}$ and see that with

$$
\sigma_{1}^{H}=\frac{b}{2}, \quad \sigma_{2}^{H} \leq 0, \quad \sigma_{3}^{H}=\frac{c}{2},
$$

we have $M_{H} \leq 0$. Note that $\Sigma_{1}^{M}$ acts on $u$ only.

The parabolic part is split into parts containing $u$ and $\mathcal{T}$ separately. For the interface condition on $u$ at $x=0$ we get by expanding (57)

$$
2 \alpha \varepsilon u_{M}\left(D_{1}^{L} u\right)_{M}+2 w_{M}^{T} \Sigma_{2}^{M} w_{M}-2 \alpha \varepsilon\left(D_{1}^{L} \mathbf{u}\right)^{T} P_{L}\left(D_{1}^{L} \mathbf{u}\right) .
$$

We choose

$$
\Sigma_{2}^{M}=\left[\begin{array}{ccc}
0 & 0 & 0 \\
0 & \sigma_{2}^{M} & 0 \\
0 & 0 & 0
\end{array}\right]
$$

and rewrite (61) as

$$
2 \alpha \varepsilon u_{M}\left(D_{1}^{L} u\right)_{M}+2 \sigma_{2}^{M} u_{M}^{2}-2 \alpha \varepsilon\left\|D_{1}^{L} \mathbf{u}\right\|_{P_{L}}^{2} .
$$

The last term in (63) can be written as

$$
\begin{aligned}
\left\|D_{1}^{L} \mathbf{u}\right\|_{P_{L}}^{2} & =\left(D_{1}^{L} \mathbf{u}\right)^{T} P_{L}\left(D_{1}^{L} \mathbf{u}\right) \\
& =\left[\begin{array}{c}
\left(D_{1}^{L} u\right)_{0} \\
\vdots \\
\left(D_{1}^{L} u\right)_{M}
\end{array}\right]^{T}\left[\begin{array}{ccc}
P_{L}^{(0,0)} & \cdots & \cdot \\
\vdots & \ddots & \vdots \\
\cdot & \cdots & P_{L}^{(M, M)}
\end{array}\right]\left[\begin{array}{c}
\left(D_{1}^{L} u\right)_{0} \\
\vdots \\
\left(D_{1}^{L} u\right)_{M}
\end{array}\right] \\
& =\left(D_{1}^{L} \mathbf{u}\right)^{T} \tilde{P}_{L}\left(D_{1}^{L} \mathbf{u}\right)+\left(D_{1}^{L} \mathbf{u}\right)_{M}^{T} p_{M}^{L}\left(D_{1}^{L} \mathbf{u}\right)_{M}
\end{aligned}
$$


where $p_{M}^{L}>0$ is such that $P_{L}^{(M, M)}-p_{M}^{L} \geq 0$. This means that the remaining matrix $\tilde{P}_{L}$ is positive semi-definite and is as such a semi-norm. Using (64) we can write (63) as

$$
\left[\begin{array}{c}
u_{M} \\
\left.\left(D_{1}^{L} u\right)_{M}\right)
\end{array}\right]^{T} \underbrace{\left[\begin{array}{cc}
2 \sigma_{2}^{M} & \alpha \varepsilon \\
\alpha \varepsilon & -2 \varepsilon p_{M}^{L}
\end{array}\right]}_{M_{u}}\left[\begin{array}{c}
u_{M} \\
\left.\left(D_{1}^{L} u\right)_{M}\right)
\end{array}\right]
$$

where $M_{u} \leq 0$ if

$$
\sigma_{1}^{u} \leq \frac{-\alpha \varepsilon}{4 p_{M}^{L}}
$$

which can be seen by computing the eigenvalues of $M_{u}$.

The remaining terms are used for coupling the two heat equations. Let the penalty matrices have the form

$$
\Sigma_{3}^{M}=\left[\begin{array}{ccc}
0 & 0 & 0 \\
0 & 0 & 0 \\
0 & 0 & \sigma_{3}^{M}
\end{array}\right], \quad \Sigma_{4}^{M}=\left[\begin{array}{ccc}
0 & 0 & 0 \\
0 & 0 & 0 \\
0 & 0 & \sigma_{4}^{M}
\end{array}\right], \quad \Sigma_{5}^{M}=\left[\begin{array}{ccc}
0 & 0 & 0 \\
0 & 0 & 0 \\
0 & 0 & \sigma_{5}^{M}
\end{array}\right],
$$

and expand the remaining terms. This gives us

$$
\begin{aligned}
& 2 \beta \varepsilon \mathcal{T}_{M}\left(D_{1}^{L} \mathcal{T}\right)_{M}-2 \beta \varepsilon\left(D_{1}^{L} \mathcal{T}\right)^{T} P_{L}\left(D_{1}^{L} \mathcal{T}\right) \\
+ & 2 \sigma_{3}^{M} \mathcal{T}_{M}\left(\mathcal{T}_{M}-T_{0}\right)+2 \sigma_{4}^{M}\left(D_{1}^{L} \mathcal{T}\right)_{M}\left(\mathcal{T}_{M}-T_{0}\right) \\
+ & 2 \sigma_{5}^{M} \mathcal{T}_{M}\left(\beta \varepsilon\left(D_{1}^{L} \mathcal{T}\right)_{M}-k\left(D_{1}^{R} T\right)_{0}\right) \\
- & 2 k T_{0}\left(D_{1}^{R} T\right)_{0}-2 k\left(D_{1}^{R} \mathbf{T}\right)^{T} P_{R}\left(D_{1}^{R} \mathbf{T}\right) \\
+ & 2 \tau_{1}^{0} T_{0}\left(T_{0}-\mathcal{T}_{M}\right)+2 \tau_{2}^{0}\left(D_{1}^{R} T\right)_{0}\left(T_{0}-\mathcal{T}_{M}\right) \\
+ & 2 \tau_{3}^{0} T_{0}\left(k\left(D_{1}^{R} T\right)_{0}-\beta \varepsilon\left(D_{1}^{L} \mathcal{T}\right)_{M}\right)
\end{aligned}
$$

which we need to bound by choosing appropriate penalty coefficients. Expression (68) can be written in matrix form as

$$
v_{I}^{T} M_{I} v_{I}-2 \beta \varepsilon\left\|D_{1}^{L} \mathcal{T}\right\|_{P_{L}}^{2}-2 k\left\|D_{1}^{R} \mathbf{T}\right\|_{P_{R}}^{2}
$$

where $v_{I}=\left[\mathcal{T}_{M},\left(D_{1}^{L} \mathcal{T}\right)_{M}, T_{0},\left(D_{1}^{R} T\right)_{0}\right]^{T}$ and

$$
M_{I}=\left[\begin{array}{cccc}
2 \sigma_{3}^{M} & \beta \varepsilon+\alpha \varepsilon \sigma_{5}^{M}+\sigma_{4}^{M} & -\left(\sigma_{3}^{M}+\tau_{1}^{0}\right) & -\left(\beta k \sigma_{5}^{M}+\tau_{2}^{0}\right) \\
\beta \varepsilon+\alpha \varepsilon \sigma_{5}^{M}+\sigma_{4}^{M} & 0 & -\left(\sigma_{4}^{M}+\alpha \varepsilon \sigma_{3}^{M}\right) & 0 \\
-\left(\sigma_{3}^{M}+\tau_{1}^{0}\right) & -\left(\sigma_{4}^{M}+\alpha \varepsilon \sigma_{3}^{M}\right) & 2 \tau_{1}^{0} & -k+\beta k \tau_{3}^{0}+\tau_{2}^{0} \\
-\left(\beta k \sigma_{5}^{M}+\tau_{2}^{0}\right) & 0 & -k+\beta k \tau_{3}^{0}+\tau_{2}^{0} & 0
\end{array}\right] .
$$

In order for the coupling terms to be bounded we need $M_{I} \leq 0$. The columns which have zero on the diagonal must be cancelled. This gives a system of equations with a one parameter family of solutions

$$
s \in \mathbb{R}, \quad \sigma_{4}^{M}=-\beta \varepsilon(1+s), \quad \sigma_{5}^{M}=s, \quad \tau_{2}^{0}=-k s, \quad \tau_{3}^{0}=1+s .
$$

Using relations (71), $M_{I}$ reduces to

$$
M_{I}=\left[\begin{array}{cccc}
2 \sigma_{3}^{M} & 0 & -\left(\sigma_{3}^{M}+\tau_{1}^{0}\right) & 0 \\
0 & 0 & 0 & 0 \\
-\left(\sigma_{3}^{M}+\tau_{1}^{0}\right) & 0 & 2 \tau_{1}^{0} & 0 \\
0 & 0 & 0 & 0
\end{array}\right]
$$

and by choosing

$$
\sigma_{3}^{M}=\tau_{1}^{0} \leq 0
$$

we have $M_{I} \leq 0$ and all coupling terms are bounded.

Using all the above we can thus conclude. 
Proposition 3.1. The schemes (32) and (33) coupled at $x=0$ are stable using the SAT boundary and interface treatment with penalty coefficients given by (41), (45), (49), (60), (66), (71) and (73).

Remark 3.3. As in the continuous case we have assumed the boundary data to be identically zero. If we would have obtained an energy estimate with non-zero data the coupled schemes would have been strongly stable [15]. No assumptions on the data at the interface have been made and hence the interface can be called strongly stable.

\section{Order of convergence}

The order of convergence is studied by the method of manufactured solutions. A small enough time step has been chosen in order to minimize the errors from the time discretization, which in this case is done by the classical 4th order explicit Runge-Kutta method. We use the functions

$$
\rho=x e^{-\kappa t}, \quad u=\sin (x) e^{-\kappa t}, \quad \mathcal{T}=\frac{1}{\varepsilon} \sin (x) e^{-\kappa t}, \quad T=\frac{1}{k} \sin (x) e^{-\kappa t}, \quad \kappa=0.1
$$

which inserted into (1) and (2) gives a modified system of equations with additional forcing functions. The functions (74) have been chosen since they satisfy the interface conditions in a non-trivial way. Using (74) we create exact initial data and time dependent boundary conditions while no data is created at the interface. The rate of convergence is obtained as

$$
q_{j}^{i}=\log _{10}\left(\frac{\left\|u_{j-1}^{i}-v_{j-1}^{i}\right\|}{\left\|u_{j}^{i}-v_{j}^{i}\right\|}\right) / \log _{10}\left(\frac{h_{j}}{h_{j-1}}\right)
$$

where $q_{j}^{i}$ denotes the convergence rate for either of the variables $i=\rho, u, \mathcal{T}, T$ at mesh refinement level $j . u_{j}^{i}$ is the exact analytic solution for either of the variables $i$ at mesh refinement level $j$ and $v_{j}^{i}$ is the discrete solution. The ratio $h_{j} / h_{j-1}$ is the ratio between the number of grid points at each refinement level. The coefficients in (1) and (2) have been chosen as

$$
a=0.5, \quad b=\frac{1}{\sqrt{\gamma}}, \quad c=\sqrt{\frac{\gamma-1}{\gamma}}, \quad \gamma=1.4, \quad \alpha=\beta=1, \quad \varepsilon=0.1, \quad k=1 .
$$

and the results are seen in Table 2 . 


\begin{tabular}{|c|c|c|c|}
\hline$M=N$ & 2nd order & 3rd order & 4th order \\
\hline & $\rho$ & $\rho$ & $\rho$ \\
\hline 32 & 0.4057 & 3.0781 & 3.4417 \\
\hline 64 & 1.9030 & 3.3046 & 3.7813 \\
\hline 128 & 1.8379 & 2.5606 & 3.8508 \\
\hline \multirow{2}{*}{256} & 2.0639 & 2.8343 & 3.8872 \\
\hline & $u$ & $u$ & $u$ \\
\hline 32 & 2.2408 & 4.5119 & 4.8983 \\
\hline 64 & 2.7097 & 3.0055 & 4.8974 \\
\hline 128 & 2.2170 & 2.9619 & 4.5113 \\
\hline \multirow[t]{2}{*}{256} & 2.1150 & 2.9809 & 4.0608 \\
\hline & $\mathcal{T}$ & $\mathcal{T}$ & $\mathcal{T}$ \\
\hline 32 & 1.4329 & 2.5140 & 3.5125 \\
\hline 64 & 2.1434 & 2.8241 & 3.6528 \\
\hline 128 & 2.0992 & 2.9815 & 3.8013 \\
\hline \multirow[t]{2}{*}{256} & 2.0649 & 3.0065 & 3.8949 \\
\hline & $T$ & $T$ & $T$ \\
\hline 32 & 2.3842 & 3.1534 & 3.9390 \\
\hline 64 & 2.1733 & 3.2604 & 3.8269 \\
\hline 128 & 2.0874 & 3.1203 & 3.8717 \\
\hline 256 & 2.0445 & 3.0500 & 3.9250 \\
\hline
\end{tabular}

Table 2: Order of convergence

The rates of convergence in Table 2 agree with the theoretically expected results $[3,6]$. The convergence in this case can be improved by using a second derivative difference operator on compact form (if the solution of the coupled problem is proven to be pointwise bounded and the penalty coefficients are chosen correctly) [16]. This case is not considered in this paper since we are aiming for the compressible Navier-Stokes equations where the diffusive terms have variable coefficients. For this type of problem the theory for the compact formulation is not yet satisfactory and work remains to be done.

\section{Spectral analysis and convergence to steady-state}

When doing flow computations one is often interested in reaching the steady state solution fast. From (32) and (33) we can see that we can write the fully coupled scheme as

$$
\frac{d \mathbf{v}}{d t}=H \mathbf{v}+F
$$

where the entire spatial discretization has been collected in the matrix $H$ and $F$ contains the boundary data. There are mainly two ways of enhancing convergence to steady-state. One is to make a spatial discretization which has negative real parts of the eigenvalues with as large magnitude as possible. That will optimize the convergence to steady-state for the ODE system $(77)[17,18,19]$. The second is to advance in time with as large time step as possible. For an explicit time integration method, the time step is limited by the eigenvalue with largest modulus.

The scheme and penalty parameters are independent of the order of accuracy of the difference operators and hence we can study the spectrum of $H$ for different orders. The first thing to be noticed is that there are two undetermined parameters $r$ and $s$ coming from the left boundary (45) and the interface (71). Theoretically any choice of these 
parameters lead to a stable scheme. With a too large magnitude they will make the problem stiff and a smaller time step is needed. Within a decent range it is interesting to see how the spectrum of $H$ changes as a function of these parameters.

In Figure 3 the minimum real part of the spectrum of $H$ is plotted as a function of $r$ and $s$ for $M=N=16$. Since the scheme is stable all real parts are negative ${ }^{1}$.

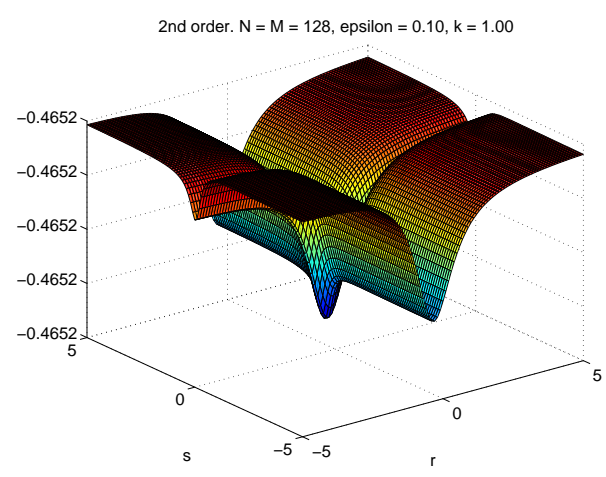

(a) 2nd order

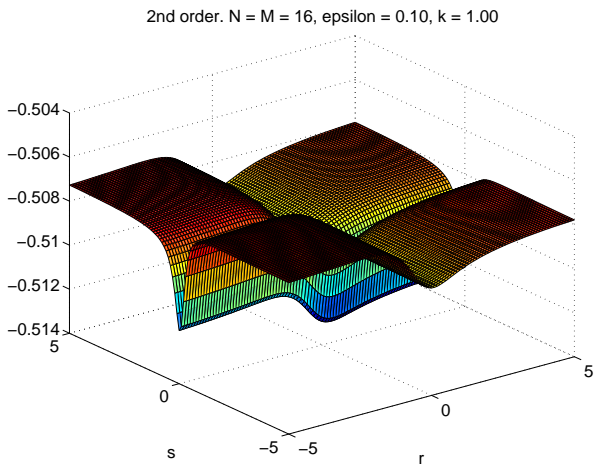

(b) 3rd order

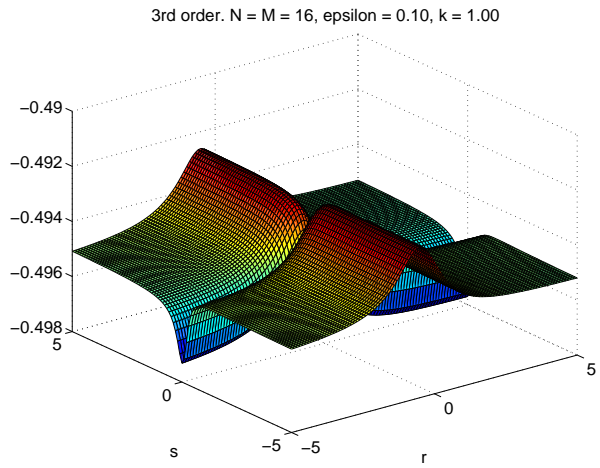

(c) 4 th order

Figure 3: Minimum real part of the eigenvalues of the spatial discretization as a function of the boundary and interface parameters $r$ and $s$ for $M=N=16$ grid points. Note that the surfaces become flatter with higher orders due to the improved convergence.

As the mesh is refined the dependence of the boundary and interface parameter disappears and the minimum real part of the eigenvalues converge to the same value for all choices and all orders of accuracy, see Figure 4.

\footnotetext{
${ }^{1}$ Minimum will refer to the minimum modulus of the real part of the spectrum. It is the eigenvalue with negative real part closest to zero which will be of our interest.
} 


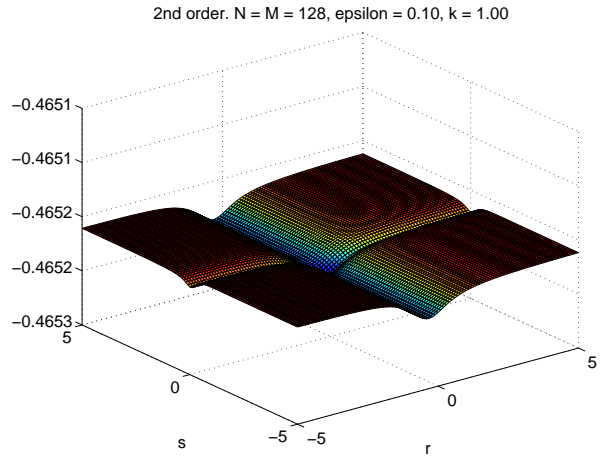

(a) 2nd order

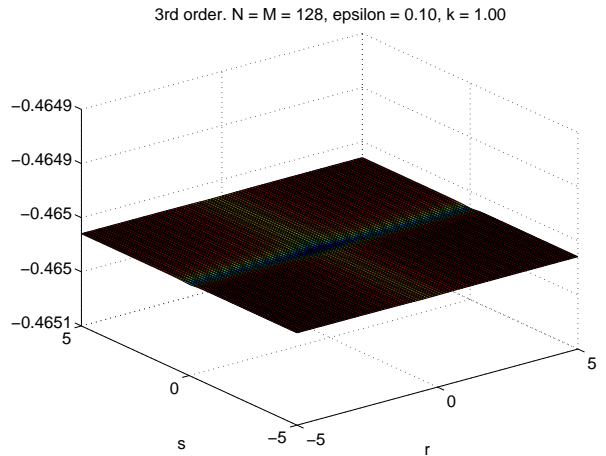

(b) 3rd order

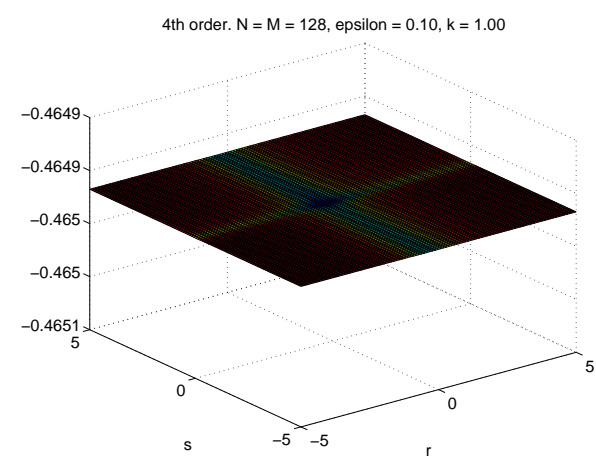

(c) 4th order

Figure 4: Minimum real part of the eigenvalues of the spatial discretization as a function of the boundary and interface parameters $r$ and $s$ for $M=N=128$ grid points

To see the convergence of the spectrum we compute the minimum real part of the eigenvalues of the spatial discretization for an increasing number of grid points. The boundary and interface parameter have been chosen as $r=-0.4$ and $s=0$ for all orders and number of grid points. The choice $r=-0.4$ makes the penalty coefficients at the left boundary to be of approximately the same magnitude. All choices of $r$ with a magnitude of order one lead to approximately the same results. The results are shown in Table 3 and Figure 5 where we can see that the minimum real part of the spectrum of the discretization converges for all orders as they should.

\begin{tabular}{|c|c|c|c|}
\hline & \multicolumn{3}{|c|}{ Minimum real part of the spectrum } \\
\hline$M=N$ & 2nd order & 3rd order & 4th order \\
\hline 16 & -0.51243 & -0.49439 & -0.48368 \\
32 & -0.47414 & -0.46904 & -0.46686 \\
64 & -0.46658 & -0.46545 & -0.46510 \\
128 & -0.46524 & -0.46502 & -0.46497 \\
256 & -0.46501 & -0.46497 & -0.46496 \\
\hline
\end{tabular}

Table 3: Minimum real part of the spectrum of the spatial discretization 


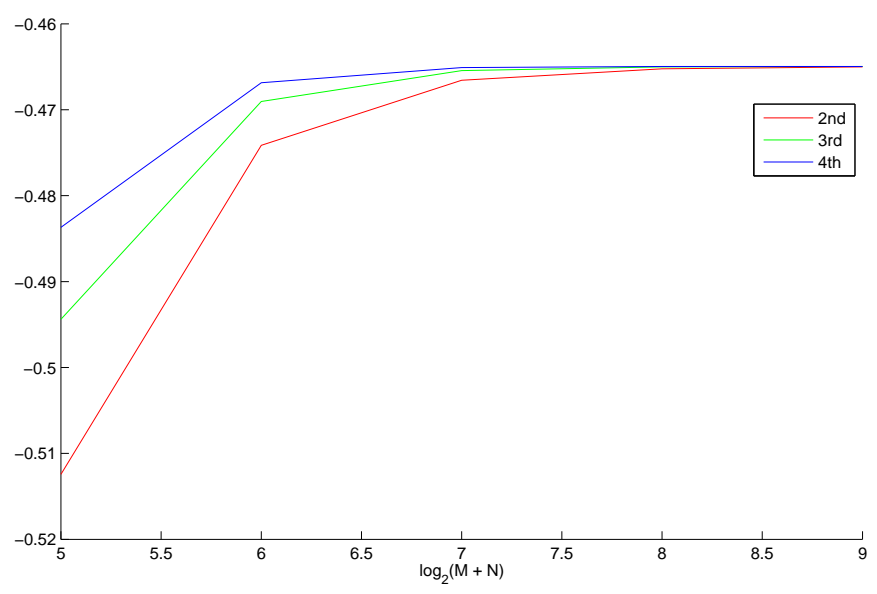

Figure 5: Convergence of the minimum real part of the discrete spectrum for 2nd, 3rd and 4th order spatial discretization

The parameter $s$ in (71) is of particular interest. In the figures and tables below we have chosen $\sigma_{3}^{M}=\tau_{1}^{0}=0$ and hence the coupling depends only on $s$. By choosing $s=0$, Dirichlet conditions for continuity of temperature are given to the fluid domain and Neumann conditions for continuity of heat flux to the solid domain. By choosing $s=-1$ we get the reversed order. By chosing $s$ such that that no terms are canceled in (50) and (51) we get a mixed type of interface conditions.

As can be seen from Figure 3 there are variations depending on the choice of $r$ and $s$ for a coarse mesh. Since we are interested in the properties of the discretization depending on the coupling, we fix $r=-0.4$ and compute the minimum real part of the spectrum as a function of $s$. The result can be seen in Table 4 .

\begin{tabular}{|c|c|c||c|c||c|c|}
\hline & \multicolumn{2}{|c||}{ 2nd order } & \multicolumn{2}{c||}{ 3rd order } & \multicolumn{2}{c|}{ 4th order } \\
\hline$M=N$ & $s$ & $\min \Re(\lambda)$ & $s$ & $\min \Re(\lambda)$ & $s$ & $\min \Re(\lambda)$ \\
\hline 16 & 0.0 & -0.51243 & 0.0 & -0.49439 & 0.0 & -0.48368 \\
32 & 0.0 & -0.47414 & 0.0 & -0.46904 & 0.0 & -0.46686 \\
64 & 0.0 & -0.46658 & 0.0 & -0.46545 & 0.0 & -0.46510 \\
128 & 0.0 & -0.46524 & 0.0 & -0.46502 & 0.0 & -0.46497 \\
\hline \hline 16 & -1.0 & -0.50873 & -1.0 & -0.49253 & -1.0 & -0.48273 \\
32 & -1.0 & -0.47367 & -1.0 & -0.46888 & -1.0 & -0.46680 \\
64 & -1.0 & -0.46651 & -1.0 & -0.46544 & -1.0 & -0.46509 \\
128 & -1.0 & -0.46524 & -1.0 & -0.46502 & -1.0 & -0.46497 \\
\hline
\end{tabular}

Table 4: The value of $s$ which give minimal real part of the spectrum is shown in the upper part. The lower part includes a comparison with the case $s=-1$.

Interface procedures for the heat equation have been considered before by eg. Giles [20], Roe et al. [21] and recently by Henshaw and Chand [22]. Giles demonstrates a method where giving Dirichlet conditions for continuity of temperature to the fluid domain and Neumann conditions for continuity of heat flux to the solid domain is necessary for preserving stability, but that the time step restriction for certain discretizations and diffusion coefficients is more severe than in each of the subdomains. Roe et al. utilizes a different discretization and is able to circumvent this restriction by deriving a set of interface equations from the interface conditions that improve the stability characteristics 
and also preserve the accuracy of the scheme. Henshaw and Chand considers many different interface procedures and prove both stability and second order accuracy independent of the diffusive properties in contrast to the results in [20]. They also state that more attractive convergence results might be obtained by considering a mixed type of interface conditions.

As can be seen from Table 4 the choice $s=0$, which correspond to the method in the above papers, is preferable since it maximizes the real part of the spectrum and hence improves the convergence. It is also clear that the difference between the results for $s=0$ and $s=-1$ are small. We investigated the intermediate values as well and not much difference in $\min \Re(\lambda)$ was found.

Regarding the issue of stiffness and the time step we can perform the same procedure as above but instead compute the maximum modulus of the spectrum as a function of $r$ and $s$. The results for $M=N=16$ grid points are shown in Figure 6 .

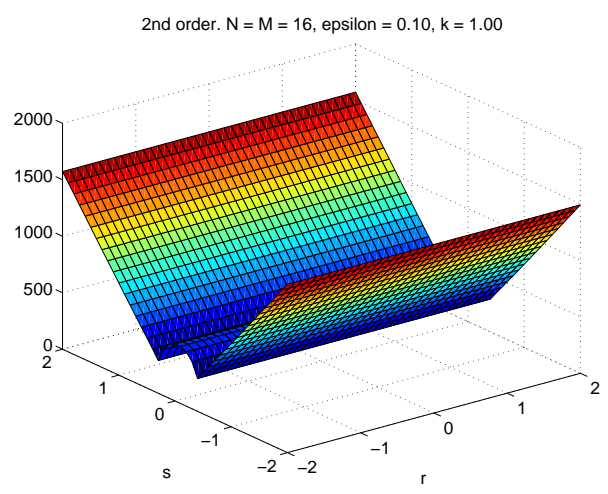

(a) 2nd order

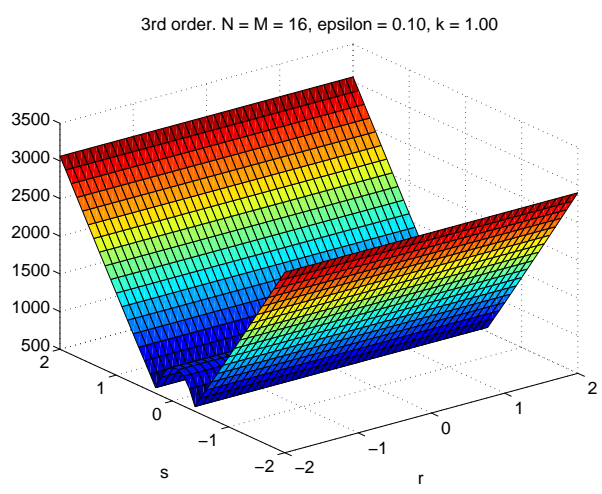

(b) 3rd order

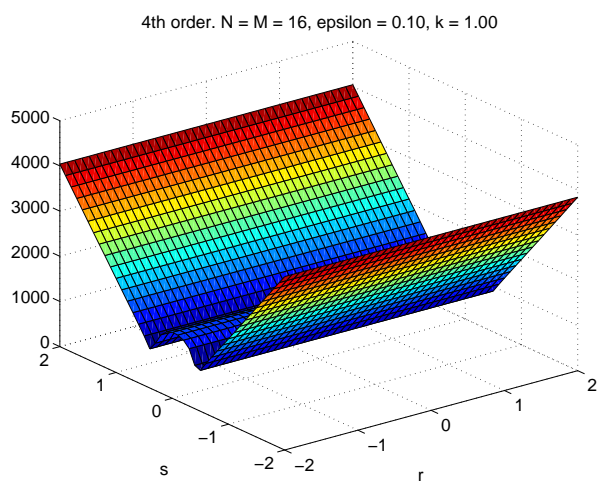

(c) 4 th order

Figure 6: Maximum absolute value of the eigenvalues of the spatial discretization as a function of the boundary and interface parameters $r$ and $s$ for $M=N=16$ grid points

Clearly the stiffless is strongly influenced by $s$ related to the interface coupling $s$ but not by $r$ relating to the left boundary condition. As before we fix $r=-0.4$ and compute the maximum modulus of the spectrum as a function of $s$. The result is seen in Table 5 together with a comparison with the extremal cases $s=0$ and $s=-1$. 


\begin{tabular}{|c|c|c||c|c||c|c|}
\hline & \multicolumn{2}{|c||}{ 2nd order } & \multicolumn{2}{c||}{ 3rd order } & \multicolumn{2}{c|}{4 th order } \\
\hline$M=N$ & $s$ & $\max |\lambda|$ & $s$ & $\max |\lambda|$ & $s$ & $\max |\lambda|$ \\
\hline 16 & 0.27 & 262.19599 & 0.26 & 499.82195 & -0.47 & 831.16460 \\
32 & 0.28 & 1048.61887 & 0.26 & 1956.94999 & -0.47 & 3321.53520 \\
64 & 0.28 & 4130.98536 & 0.26 & 7730.09701 & -0.47 & 13286.14080 \\
128 & 0.28 & 16385.83220 & 0.26 & 30875.13265 & -0.47 & 53144.56320 \\
\hline \hline 16 & 0.0 & 506.10401 & 0.0 & 946.87926 & 0.0 & 1461.33389 \\
32 & 0.0 & 2023.97316 & 0.0 & 3787.78434 & 0.0 & 5845.48940 \\
64 & 0.0 & 8095.61354 & 0.0 & 15151.67511 & 0.0 & 23382.27042 \\
128 & 0.0 & 32382.50222 & 0.0 & 60607.77891 & 0.0 & 93529.71064 \\
\hline \hline 16 & -1.0 & 736.43767 & -1.0 & 1427.31703 & -1.0 & 1889.05321 \\
32 & -1.0 & 2941.31462 & -1.0 & 5703.41160 & -1.0 & 7548.91634 \\
64 & -1.0 & 11756.61529 & -1.0 & 22801.96629 & -1.0 & 30181.10326 \\
128 & -1.0 & 47009.40113 & -1.0 & 91184.53704 & -1.0 & 120695.31884 \\
\hline
\end{tabular}

Table 5: The values of $s$ which gives minimum largest modulo of the spectrum is shown in the upper part. The lower parts includes a comparison with the cases $s=0$ and $s=-1$.

As can be seen in Table 5 the stiffness can be reduced by choosing a mixed type of interface condition, and hence bigger time steps can be used. Compared to the extremal values $s=0$ and $s=-1$ the optimal choices of $s$ allows one to take almost twice as big time step and maintain stability for an explicit time integration method. This result is discussed in [22] for the heat equation and we can now verify it for this more general problem.

\section{An application}

An example of a solution, where the coefficients are given by (76) is given in Figure 7 . We start with zero initial data and at time $t=0$ we let $\rho=0, u=0.5$ and $\mathcal{T}=1$ at the left boundary while $T=0$ at the right boundary. The values at the left boundary is transformed into data for the characteristic boundary conditions. 

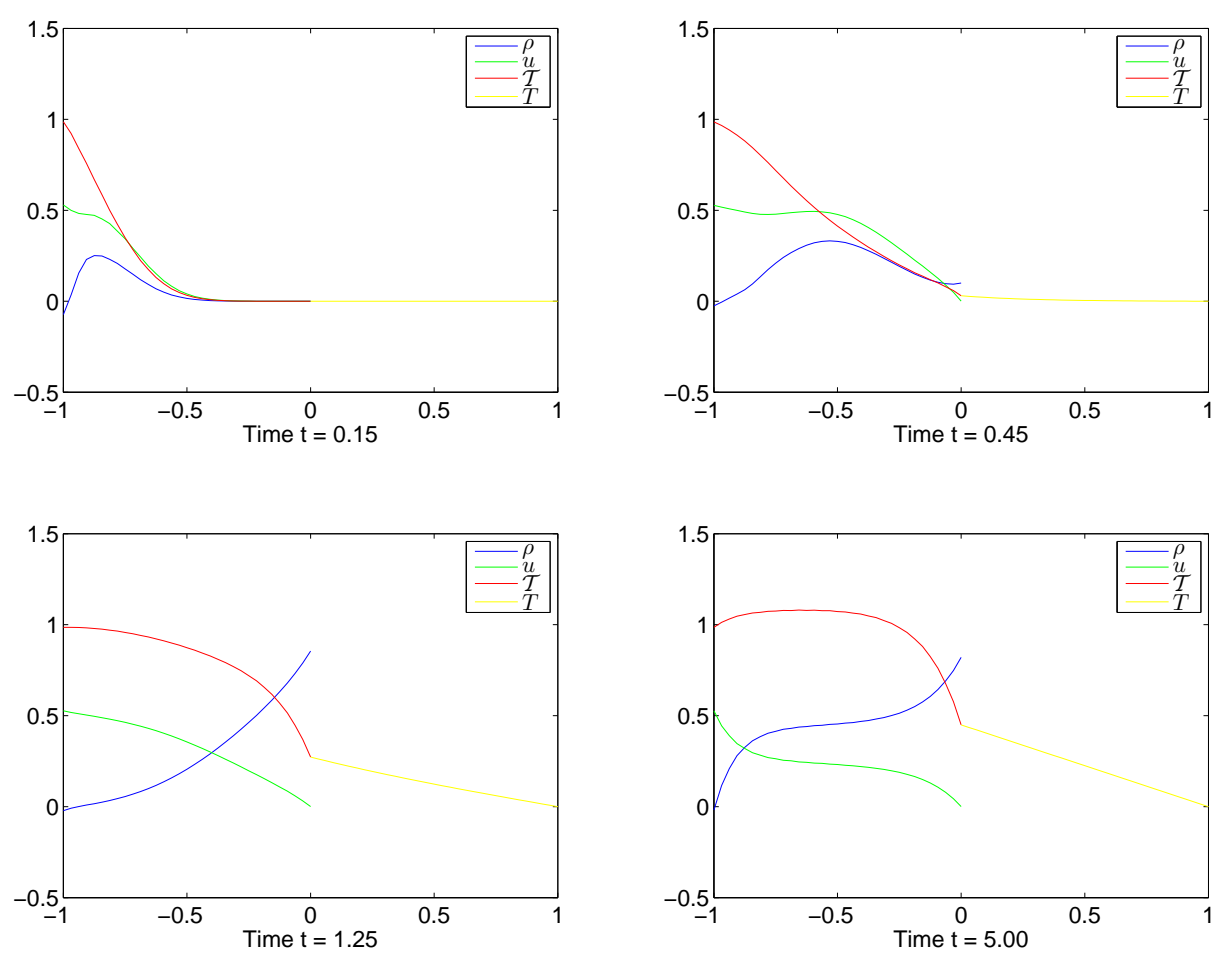

Figure 7: A sequence of coupled solutions for different times using $M=N=32$ grid points and 3rd order operators. The last figure shows the steady state solution where the ratio $\mathcal{T}_{x} / T_{x}$ of the heat fluxes at the interface is exactly the ratio $k / \varepsilon$ of the diffusion coefficients.

To see why the coupling is necessary we give a comparison where the interface conditions have been replaced by attempting to give boundary conditions to the separate equations. At the left and right boundaries we give the same boundary data. At the interface we give $\mathcal{T}=T=0$ for continuity and $u=0$. Since the left equation is the heat equation with zero boundary data once has no reason to give anything else. The result is seen in Figure 8. 

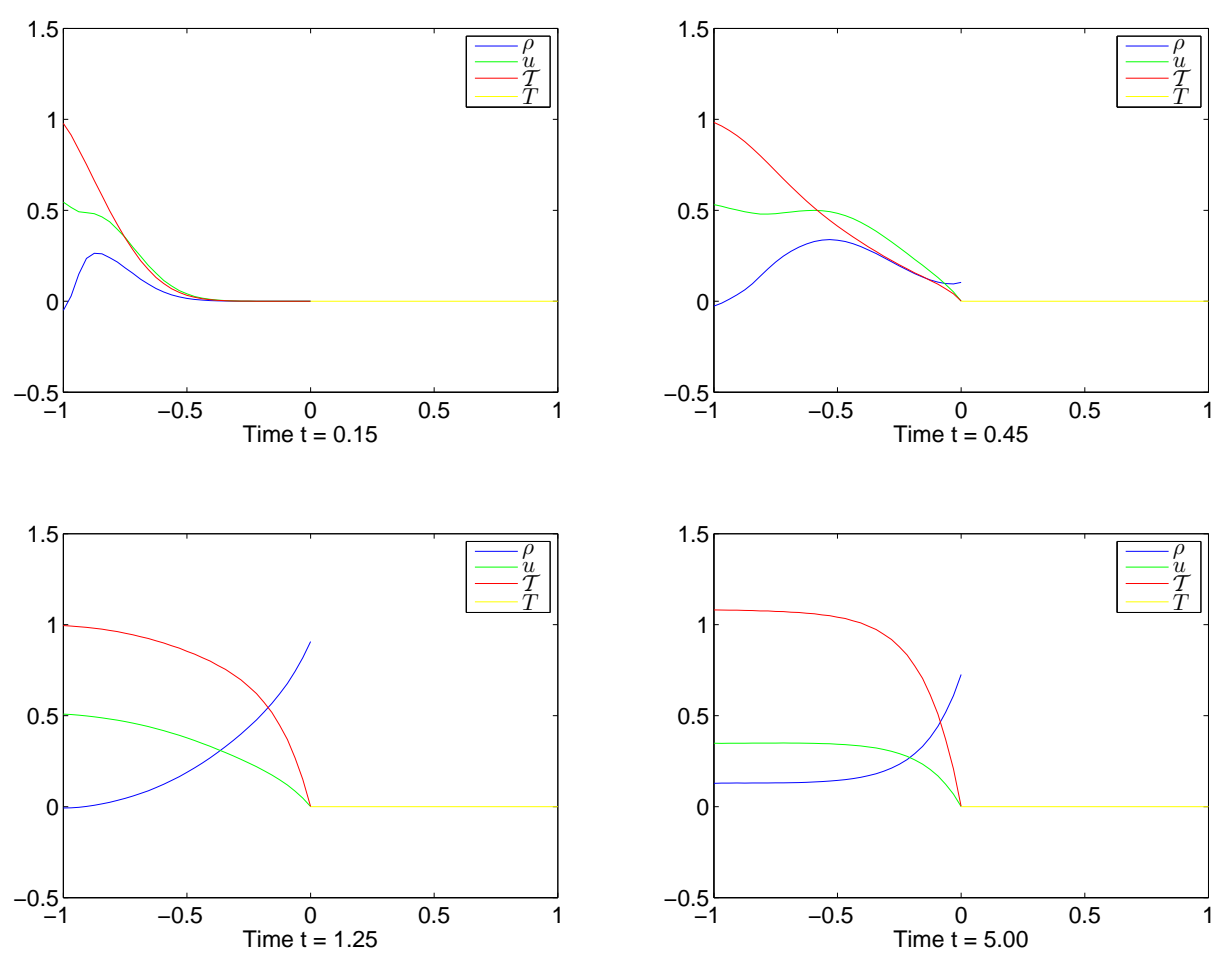

Figure 8: A sequence of uncoupled solutions for different times using $M=N=32$ grid points and 3rd order operators

We can clearly see that for $t=0.15$ and $t=0.45$ the solutions are similar, but as soon as the influences from the left boundary reaches the interface the solutions start to deviate. The steady-state solution is significantly different not only for the coupled variables.

\section{Summary and conclusions}

An incompletely parabolic system of equations is coupled with the heat equation in one space dimension. The energy method is used to derive well-posed boundary and interface conditions. The equations are discretized using finite differences on Summation-by-Parts form where the boundary and interface conditions are weakly imposed using the Simultaneous Approximation Term. The penalty matrices and coefficients are determined such that the boundaries are stable and the interface can be called strongly stable.

The interface conditions are derived such that we can study different interface conditions as a function of one parameter. By looking at the spectrum of the spatial discretization as a function of the interface parameter, it can be seen that there are only minor differences between the minimun real part of the spectrum for different coupling techniques. However when giving a mixed type of interface condition the stiffness is greatly reduced and an almost twice as big time step can be used while maintaining stability for an explicit time integration method.

The rate of convergence is verified by the method of manufactured solutions and the result is consistent with the theory within the SBP framework. The derived numerical schemes are independent of the order of accuracy and higher order accuracy is easily obtained by using difference operators of higher orders. An example where the system is 
solved using 3rd order operators is shown and it can be seen that the correct interface conditions are obtained.

\section{References}

[1] Jens Lindström, Johan Bejhed, and Jan Nordström. Measurements and numerical modelling of orifice flow in microchannels. In the 41st AIAA Thermophysics Conference, AIAA Paper No. 2009-4098, San Antonio, USA, 22-25 June 2009.

[2] Bo Strand. Summation by Parts for Finite Difference Approximations for d/dx. Journal of Computational Physics, 110(1):47 - 67, 1994.

[3] Ken Mattsson and Jan Nordström. Summation by parts operators for finite difference approximations of second derivatives. Journal of Computational Physics, 199(2):503$540,2004$.

[4] Ken Mattsson. Boundary Procedures for Summation-by-Parts Operators. J. Sci. Comput., 18(1):133-153, 2003.

[5] Mark H. Carpenter, Jan Nordström, and David Gottlieb. A Stable and Conservative Interface Treatment of Arbitrary Spatial Accuracy. Journal of Computational Physics, 148(2):341 - 365, 1999.

[6] Jing Gong and Jan Nordström. Stable, Accurate and Efficient Interface Procedures for Viscous Problems. Report, Uppsala University, Disciplinary Domain of Science and Technology, Mathematics and Computer Science, Department of Information Technology, Numerical Analysis, Department of Information Technology, Uppsala University, Uppsala, 2006.

[7] X. Huan, J.E. Hicken, and D.W. Zingg. Interface and Boundary Schemes for HighOrder Methods. In the 39th AIAA Fluid Dynamics Conference, AIAA Paper No. 2009-3658, San Antonio, USA, 22-25 June 2009.

[8] Magnus Svärd, Mark H. Carpenter, and Jan Nordström. A stable high-order finite difference scheme for the compressible Navier-Stokes equations, far-field boundary conditions. J. Comput. Phys., 225(1):1020-1038, 2007.

[9] Magnus Svärd and Jan Nordström. A stable high-order finite difference scheme for the compressible Navier-Stokes equations: No-slip wall boundary conditions. Journal of Computational Physics, 227(10):4805 - 4824, 2008.

[10] Ken Mattsson, Magnus Svärd, Mark Carpenter, and Jan Nordström. High-order accurate computations for unsteady aerodynamics. Computers and Fluids, 36(3):636 $-649,2007$.

[11] Jan Nordström, Jing Gong, Edwin van der Weide, and Magnus Svärd. A stable and conservative high order multi-block method for the compressible Navier-Stokes equations. Journal of Computational Physics, 2009.

[12] Ken Mattsson, Magnus Svärd, and Jan Nordström. Stable and Accurate Artificial Dissipation. J. Sci. Comput., 21(1):57-79, 2004.

[13] Jan Nordström and Magnus Svärd. Well-Posed Boundary Conditions for the NavierStokes Equations. SIAM J. Numer. Anal., 43(3):1231-1255, 2005. 
[14] Saul Abarbanel and David Gottlieb. Optimal time splitting for two- and threedimensional Navier-Stokes equations with mixed derivatives. Journal of Computational Physics, 41(1):1 - 33, 1981.

[15] Bertil Gustafsson, Heinz-Otto Kreiss, and Joseph Oliger. Time Dependent Problems and Difference Methods. Wiley Interscience, 1995.

[16] Magnus Svärd and Jan Nordström. On the order of accuracy for difference approximations of initial-boundary value problems. Journal of Computational Physics, 218(1):333-352, 2006.

[17] Magnus Svärd, Ken Mattsson, and Jan Nordström. Steady-State Computations Using Summation-by-Parts Operators. J. Sci. Comput., 24(1):79-95, 2005.

[18] Jan Nordström. The influence of open boundary conditions on the convergence to steady state for the Navier-Stokes equations. Journal of Computational Physics, $85(1): 210-244,1989$.

[19] Björn Engquist and Bertil Gustafsson. Steady state computations for wave propagation problems. Mathematics of Computation, 49(179):39-64, 1987.

[20] M. B. Giles. Stability analysis of numerical interface conditions in fluid-structure thermal analysis. International Journal for Numerical Methods in Fluids, 25:421436, August 1997.

[21] B. Roe, R. Jaiman, A. Haselbacher, and P. H. Geubelle. Combined interface boundary condition method for coupled thermal simulations. International Journal for Numerical Methods in Fluids, 57:329-354, May 2008.

[22] William D. Henshaw and Kyle K. Chand. A composite grid solver for conjugate heat transfer in fluid-structure systems. Journal of Computational Physics, 228(10):3708 - 3741, 2009. 